\title{
Optimal CHP Planning in Integrated Energy Systems considering Network Charges
}

\author{
Hantao Wang, Student Member, IEEE, Chenghong Gu, Member, IEEE, Xin Zhang, Member, IEEE \\ and Furong Li, Senior Member, IEEE ${ }^{l}$
}

\begin{abstract}
This paper proposes a novel optimal planning model for combined heat and power (CHP) in multiple energy systems of natural gas and electricity to benefit both networks by deferring investment for network owners and reducing use-of-system (UoS) charge for network users. The new planning model considers the technical constraints of both electricity and natural gas systems. A two-stage planning approach is proposed to determine the optimal site and size of CHPs. In the first stage, a long-run incremental cost (LRIC) matrix is designed to reflect CHP locational impact on both natural gas and electxaricity network investment, used as a criterion to choose the optimal location. In the second stage, CHP size is determined by solving an integrated optimal model with the objective to minimize total incremental network investment costs. The proposed method is resolved by the interior-point method and implemented on a practically integrated electricity and natural gas systems. Two case studies are conducted to test the performance for single and multiple CHPs cases. This work can enable costefficient CHP planning to benefit integrated natural gas and electricity networks and network users in terms of reduced network investment cost and consequently reduced UoS charges.
\end{abstract}

Index Terms-Combined heat and power (CHP), optimal planning, multiple energy networks, incremental cost.

\section{NOMENCLATURE}

\begin{tabular}{|c|c|}
\hline CHP & Combined Heat and Power \\
\hline UoS & Use-of-System \\
\hline LRIC & Long run incremental cost \\
\hline $\mathrm{IC}$ & Incremental cost \\
\hline$I_{L R I C}$ & LRIC index \\
\hline$x$ & $\begin{array}{l}x^{\text {th }} \text { point coupling in integrated energy } \\
\text { networks with a total number of } X\end{array}$ \\
\hline$E$ & Electricity output (MW) \\
\hline$G$ & Gas input (MW) \\
\hline$m$ & $\begin{array}{l}m^{t h} \text { busbar of the electricity network with a } \\
\text { total number of } M\end{array}$ \\
\hline$n$ & $\begin{array}{l}n^{t h} \text { line of the electricity network with a } \\
\text { total number of } N\end{array}$ \\
\hline$S$ & $\begin{array}{l}S^{t h} \text { node of the gas network with a total } \\
\text { number of } S\end{array}$ \\
\hline$t$ & $\begin{array}{l}t^{t h} \text { pipeline of the gas network with a total } \\
\text { number of } T\end{array}$ \\
\hline$C$ & Line/pipeline capacity $\left(\mathrm{MW} / \mathrm{m}^{3} / \mathrm{s}\right)$ \\
\hline$P$ & Line active power flow (MW) \\
\hline
\end{tabular}

Hantao Wang, Chenghong Gu (corresponding author) and Furong $\mathrm{Li}$ are with the Department of Electronic \& Electrical Engineering, University of Bath, Bath, UK (H.Wang@bath.ac.uk, C.Gu@bath.ac.uk, eesfl@bath.ac.uk). Xin Zhang (xin.zhang@cranfield.ac.uk) is with the School of Water, Energy and Environment, Cranfield University, Cranfield, UK.
$Q$

$q$

$r$

$y$

$P V$

$d$

AV

$\Delta$

$A F$

$L S$

num

$G$

$B$

$G_{\text {sh }}$

$a$

V

$\theta$

Gen

Load

Flow

$V_{\min }$ and $V_{\max }$

$p$

$T$

Ef

$g$

L

Z

$D$

seg

$q_{\min }$ and $q_{\max }$

$\eta$

HtER

$W i$

$p_{\min }$ and $p_{\max }$
Line reactive power flow (MVar)

Pipeline gas flow $\left(\mathrm{m}^{3} / \mathrm{s}\right)$

Annual load growth rate (\%)

Year for current flow to reach line capacity

with load growth $r$ (years)

Present value of future investment $(\mathfrak{f})$

Discount rate (\%)

Current asset value (\%)

Difference

Annuity factor

Life span (year)

Number of CHP from 1 to $\mathrm{Num}$

Conductance

Susceptance

Shunt admittance

Tap ratio of transformer

Voltage magnitude

Voltage angel difference

Generation (MW)

Demand (MW)

Outgoing power flow (MW)

Lower and upper bound of voltage

Pipeline pressure $(\mathrm{kPa})$

Pipeline temperature $\left({ }^{\circ} \mathrm{C}\right)$

Pipeline efficiency

Gas gravity

Pipeline length $(\mathrm{km})$

Gas compressibility factor

pipeline inside diameter $(\mathrm{cm})$

Pipeline flow direction (1 or -1 )

Lower and upper bound of gas flow

Efficiency

Heat to electricity ratio

Isothermal work done, $\mathrm{J} / \mathrm{kg}$ of gas

Lower and upper bound of gas pressure

\section{INTRODUCTION}

$\mathrm{W}$ ITH the increasing penetration of gas-fired distributed generation (DG) and promising usage of power-to-gas (P2G) technology, the interdependence between electricity and natural gas networks is becoming stronger. The widespread utilisation of high efficient Combined Heat and Power (CHP) will significantly affect energy system planning, operation and 
trading in multi-carrier energy systems.

CHP or cogeneration is highly efficient to generate both electricity and heating at the same time. Compared to the conventional power plant and heating boiler, $\mathrm{CHP}$ has relatively high efficiency up to $90 \%$ as well as can reduce carbon emissions by $30 \%$ [1]. Moreover, it saves energy bills by $20 \%$ compared to typical energy consumption [2]. According to the latest Digest of UK Energy Statistics (DUKES) [3], the good quality CHP capacity in the UK of 2016 was $5571 \mathrm{MWe}$ which produced $20.1 \mathrm{TWh}$ electricity that accounted for $6.3 \%$ of all electricity supplied in the UK. With the increasing penetration of on-site CHPs, their optimal planning should not only consider technical operability and carbon emissions but also optimise network investment costs and Use of System (UoS) charges to benefit both network owners and users.

The remainder of this paper is organised as follows: the overview of the proposed model is described in Section II. Section III provides an overview of the method. The detailed optimal CHP planning of selecting site and size are elaborated in Sections IV. The electricity and natural gas network models are developed in Sections V. CHP modelling is illustrated in Section VI. Section VII demonstrate the model and Section VIII provide discussions. Conclusions are drawn in Section IX.

\section{LITERATURE REVIEW}

The majority of research on CHP planning shares commonly used optimisation objectives, such as energy savings, cost and carbon emissions reductions. In [4], the authors select the locations and size of CHP-based distributed energy resources (DER). It firstly proposes a loss sensitivity index to determine the site to minimize system losses and then uses particle swarm optimization to obtain the optimal size of CHP. In [5], CHPs are used to resolve wind power curtailment, where a new method is proposed to minimize the total generation cost of a multi-regional network. Samaneh et al. [6] proposes a method for CHP planning with several criteria, including network reliability, power losses and voltage profiles. These factors are represented by reliability cost, power losses and voltage penalty cost contained in the objective function. Paper [7] proposes an expansion planning of CHP in an energy hub system with multiple energy infrastructures. Another research in [8] analyses the practical procedure for CHP planning to determine efficiency while satisfying requirements. It also investigates the impact of a changeable practical CHP operating mode. Paper [9] introduces an optimal configuration of CHP plants with a battery energy storage to provide peak shaving service. It optimally maximises the primary energy savings while minimising the payback for both CHP and storage system. The optimal allocation proposed in [10] is focused on distribution networks with the objective to maximise electrical output and recover thermal output. The designed system is capable to recover the exhaust heat by using CHP.

Most CHP planning only considers electricity network security and the benefits of reducing electricity network investment. Nevertheless, the interdependence between the electricity and gas networks should be carefully modelled in planning CHPs. In [11], a multi-linear probabilistic energy flow of integrated electricity and natural gas systems is proposed to investigate the impact of massive uncertainties on the security and economical operation of both systems. The work in [12] also assesses an integrated energy system to achieve a lowcarbon objective, where the interconnection between electricity, gas and heating systems enabled by renewable sources allows a holistic optimal power flow assessment. Another optimal expansion planning for multiple energy infrastructures is discussed in [7]. It proposes an energy hub planning model including CHP and presents the optimal operation of the hub to achieve the least cost in planning. The planning framework for a CHP system with a solar-powered heat pump is proposed in [13] considering a bi-level market. In [14], a CHP based district heating system with renewable energy and energy storage is modelled and optimised. It proposes a planning and operation method to minimize the overall cost of the net acquisition for heat and power. Shaneb et. al [15] investigates CHP sizing for both electricity and heating to minimize expected annual cost using a generic deterministic linear programming model. A non-sequential probabilistic production simulation method of CHP planning is introduced for wind energy curtailment, considering the seasonal heat supply constraints [16]. CHP is also used in planning energy hub system, and [17] proposes an optimal integrated sizing and planning of hubs with midsize/large CHP units considering supply reliability. Paper [18] develops a CHPbased micro-grid with reserve capacity to demonstrate the economic benefit to distribution system operators.

In the UK, all network users pay UoS charges for using networks [19]. Network charges are generally used to recover the network capital, operation and maintenance costs and provide users with a forward-planning, economic-efficient economic signal. Power supplier purchases electricity and gas from generation companies on behalf of the customers. Suppliers are charged by network operators and suppliers pass these charges to customers in terms of UoS charges. These network charges account for around $13-15 \%$ of the overall bill for a typical customer. Investment cost-related pricing (ICRP) is a typical method for transmission network charges [20] and the distribution reinforcement model (DRM) [21] is developed for low voltage networks. Long run marginal cost (LRMC) [22] and long-run incremental cost (LRIC) [23, 24] are two typical methods for distribution network pricing.

Natural gas network charges can be divided into two parts: transmission system charges and distribution network charges. Transmission charging statement in [25] includes capacity charges, interconnection point capacity charges commodity charges and other charges. Gas distribution network (GDN) charges, also known as Local Distribution Zone (LDZ) charges, are levied by GDN operators to recover their regulated allowed revenue determined through the price control [26, 27]. GDN charges are paid by the gas shipped on behalf of customers. GDN charges methodologies are required to develop to achieve certain objectives including cost reflective, facilitate competition, and reflect developments in GDN businesses. With the penetration of on-site CHPs and other distributed energy resources, these energy techniques will have a profound 
impact on network charging for both electricity and gas networks. This is because that CHPs change the supply, demand and power/gas flows in both networks, which further affect network maintenance cost and investment cost and network user use-of-system charges.

By far, limited research attempts to plan CHPs considering the impact of both electricity and natural gas networks. The installation of CHP would have a significant effect on network users that pay future network investment in terms of network charges. Proper planning of CHP in the integrated electricity and gas network will benefit both network owners and users in terms of investment deferral and network charge reduction.

The objective of this paper is to optimally site and size CHPs in the integrated electricity and natural gas systems. The ultimate aim is to reduce network investment for network operators and UoS charges for network users. The proposed method is decomposed in two stages to realize the planning objective, where the first step determines the optimal locations of CHPs in the integrated network and the second step decides the optimal capacity of CHPs to be installed. To realize the objectives, a novel approach for sitting and optimization model for sizing is designed with the constraints of both systems.

The proposed method hypothetically assumes that in the integrated energy system, both electricity and gas load are growing at an annual growth rate. Therefore, the two systems will need reinforcement when their capacities are breached, which not only trigger investment costs but also consequently UoS charges for network users. With optimally planned CHPs that bridge the two systems, their reinforcement horizons could be further deferred. Therefore, the investment costs could be saved for network operators and UoS charges could be reduced for network users. In addition, it is assumed that CHPs can only be sitted at the coupling points of the two systems that are geographically close. It is to ensure that no large-scale network investment is needed to enable CHP operation.

The proposed method in this paper is decomposed into a twostage method: to determine the optimal locations of CHPs in the system and then determine their optimal capacity. Therefore, there is one objective in each step. Firstly, incremental costs are determined for electricity and natural gas network respectively, evaluated by comparing the present value difference of future reinforcement with and without CHP integration. This is achieved by assuming a unit-size CHP is installed as each available location. Then, an LRIC matrix is designed, where the coupling points between the two systems with minimum incremental costs are the potential installing sites. The sizing problem is modelled as optimisation with the objective to maximally reduce the incremental cost for both systems. In both stages, the objective function is dependent on the change of the energy flows in the integrated energy system, which is essentially caused by the installation of CHP. The proposed model is resolved by the interior-point method and then tested on a multi-carrier energy network of 15 electrical bus and 12 gas nodes. The effectiveness of single and multiple CHPs planning in reducing incremental cost (IC) are demonstrated. Results show that single CHP planning can reduce IC by $42 \%$, while the multiple CHPs planning can reduce IC further by

\begin{tabular}{|c|c|c|c|c|}
\hline \multicolumn{3}{|c|}{ Stage 1} & \multicolumn{2}{|l|}{ Stage 2} \\
\hline Variable CHP & Determine & Fixed CHP & Determined & Fixed CHP \\
\hline locations & CHP locations & locations & CHP locations & locations \\
\hline Variable CHP & & Variable CHP & Determine & Fixed CHP \\
\hline power ratings & & power ratings & CHP power rati & power ratings \\
\hline
\end{tabular}

Fig. 1. Two-stage decomposition of the proposed optimal CHP planning.

$63 \%$. The method is beneficial to both system owners and users to reduce IC in both electricity and gas networks.

The main contributions are: i) it proposes a novel planning model for CHPs in the integrated energy system of natural gas and electricity, which consider the impact of CHP on both systems. It overwhelms the existing research which only considers either electricity or natural gas networks; ii) A new LRIC index matrix is designed which easily sites CHPs on the coupling points of the two systems. It can find locations that reduce network investment costs for both systems and eventually reduce the use-of-system charges for network users; iii) It develops a new optimisation model to determine the size of CHP in the integrated electricity and natural gas systems to benefit both networks and network users considering the physical constraints of both systems

\section{OVERVIEW OF THE PROPOSED METHOD}

In this section, a two-stage solution for CHP planning in a multi-carrier energy system is proposed in Fig.1. The objective is to minimize network investment cost and consequently UoS charges for both electricity and natural gas networks. It is achieved by a two-stage solution: the first stage is to determine the site of CHP in the coupled electricity and gas networks, and the second stage is to determine CHP capacity.

Stage 1: In order to determine the locations of CHP, an LRIC matrix is built. It is derived by hypothetically placing a unit-size CHP at each coupling point between an electricity bus and a gas node once at a time. Then, the potential benefit that a CHP would bring along in terms of reducing investment costs is quantified. In this way, the LRIC matrix can reflect CHP's economic impact on both electricity and gas networks in terms of future network reinforcement deferral or reduction. Those coupling points with minimum LRIC values, which indicate the lowest network investment cost, are chosen to place the CHP.

Stage 2: the objective of sizing CHP is to minimize the total future investment cost for reinforcing both electricity and gas networks with the sites determined at Stage 1. The sizing problem is formulated as an optimization to further reduce investment costs and network charges. Constraints for electricity and natural gas networks are applied in both stages

\section{CHP SITING AND SIZING FoRMULATION CONSIDERING UOS CHARGES}

This section determines the optimal location and capacity of CHP in an integrated energy system. An LRIC matrix is formulated as an index to determine the potential sites that couple electricity and natural gas network by installing CHP. It is achieved by hypothetically adding a unit size CHP at each potential coupling point and calculating the change in the total incremental cost of both energy networks. 
For every unit CHP at each coupling point, an LRIC index is obtained to form an LRIC matrix. The point that with higher negative LRIC value indicates the greater potential of installing CHP to defer network reinforcement (investment) costs and consequently network charges. The LIRC index matrix $I_{\text {LRIC }}$ is

$$
I_{L R I C}=\left(\begin{array}{c}
L R I C_{1} \\
\vdots \\
L R I C_{x} \\
\vdots \\
L R I C_{X}
\end{array}\right)
$$

These sites between electricity and natural gas networks should be geographically close to each other, otherwise, it would be practically infeasible. $L R I C_{x}$ is the long run incremental cost assuming a unit size CHP installed at point $x$, which is the sum of the electricity network $L R I C_{E}$ and natural gas network $L R I C_{G}$. They are calculated by the following steps.

\section{A. Calculation of Electricity Network LRIC}

1) The present value of the network components

For a $M$-busbar network consisting of $N$ lines, each of the lines usually has an individual capacity to support a power flow. For a given annual load growth rate $\mathrm{p}$, the line $n$ will take years for the current power flow to reach its capacity, which is expressed in (2)

$$
C_{E_{\_} n}=P_{n} \times\left(1+r_{E_{-} n}\right)^{y_{E_{-} n}}
$$

Rearranging this equation leads to

$$
\left(1+r_{E_{-} n}\right)^{y_{E_{-} n}}=\frac{C_{E_{-} n}}{P_{n}}
$$

Taking the logarithm of both sides of (3) and rearranging it gives the value of $y_{E_{-} n}$

$$
y_{E_{-} n}=\frac{\log C_{E_{-} n}-\log P_{n}}{\log \left(1+r_{E_{-} n}\right)}
$$

Therefore, it is reasonable to assume that reinforcement will take place when the power flow $P_{n}$ on the $n^{\text {th }}$ line reaches its capacity $C_{E_{\_} n}$ after $y_{E_{\_} n}$ years.

2) The present value of future investment cost

The future investment is expressed mathematically with the current equivalent asset value and a discount rate. For simplicity, the present value of future investment cost is usually discounted back to its present value. For a given discount rate of $d_{E_{-} n}$ for the $n^{\text {th }}$ line, the present value of the future investment in year $y_{E_{-} n}$ will be:

$$
P V_{E_{-} n}=\frac{A V_{E \_n}}{\left(1+d_{E \_n}\right)^{y_{E_{-} n}}}
$$

3) The present value of the components with $C H P$

If one or several CHPs are installed in the network, apparently power flow will change along lines. To calculate the LRIC index, it is assumed that 1 unit-size electricity output $1 \mathrm{MW}$ of CHP is placed at the coupling point $x^{\text {th }}$, which is geographically at $m^{\text {th }}$ bus. For the $n^{\text {th }}$ line, its new power flow becomes $P_{\_} n e w_{E_{-} n}$. This will also result in a change of future investment horizon from $y_{E_{n} n}$ to $y_{-} n e w_{E_{-} n}$.

$$
C_{E_{-} n}=P_{-} n e w_{n} \times\left(1+r_{E_{-} n}\right)^{y_{-} n e w_{E_{-}} n}
$$

Where $P_{-} n e w_{n}$ is the new power flow along the $n^{\text {th }}$ network component caused by the unit size CHP power injection $E_{C H P}$ located at coupling point $x$. For simplicity, it is

$$
P_{-} \operatorname{new}_{n}\left(E_{C H P}\right)=P_{n}+\Delta P_{n}
$$

The new time horizon of the future investment for the $n^{\text {th }}$ line thus is

$$
y_{-} n e w_{E \_n}\left(E_{C H P}\right)=\frac{\log C_{E \_n}-\log P \_n e w_{n}}{\log \left(1+r_{E \_n}\right)}
$$

The new investment cost with the installation of CHP is

$$
P V_{-} n e w_{E_{-} n}\left(E_{C H P}\right)=\frac{A V_{E_{-} n}}{\left(1+d_{E_{-} n}\right)^{y_{-} n e w_{E_{-}} n}}
$$

The difference between the old present value and new present values of the future investment cost is given by (9)

$$
\Delta P V_{E_{-} n}\left(E_{C H P}\right)=P V_{-} n e w_{E_{-} n}-P V_{E_{-} n}
$$

For line $n$, if $\Delta P V_{E_{-} n}$ value is negative, it indicates that the installed CHP benefits this asset by reducing loading, extending the future reinforcement horizon, and eventually reducing the cost of the future investment that network users need to pay in terms of network charges.

\section{4) The Change of LRIC with CHP}

Annuity Factor $A F$ is introduced here to reflect the time value of money. This factor shows the ratio between a series of regular payments/income and future payment/income, formulated in (11).

$$
A F_{E_{-} n}=\frac{1-\left(1+d_{E_{-} n}\right)^{-L S_{E_{-} n}}}{d_{E_{-} n}}
$$

By dividing $\triangle P V_{E_{-} n}$ with $A F_{E_{-} n}$, then dividing again by the unit size of the CHP electricity output at $m^{t h}$ busbar (which is $x^{\text {th }}$ point to couple multiple energy networks), this model produces LRIC that reflects CHP impact on the $n^{\text {th }}$ line. Therefore, the overall LRIC of all $N$ line of this electricity network with a unit power output CHP at $x^{\text {th }}$ coupling point is:

$$
L R I C_{E_{-} x}=\sum_{n=1}^{N} \frac{\Delta P V_{E_{\_} n}\left(E_{C H P}\right)}{E_{C H P} \times A F_{E_{-} n}}
$$

According to (1) to (12), the change of $L R I C_{E_{-} x}$ depends on the change of $\Delta P_{n}$, which is caused by CHP injection at $x^{\text {th }}$ coupling point in the integrated energy network.

\section{B. Calculation of Natural Gas Networks LRIC}

The formulation steps of LRIC for natural gas networks are similar to that of electricity network described in Section A. For a $S$-node natural gas network with $T$ pipelines, if the pipeline configuration is fixed, the gas flow along each pipeline is usually decided by the inlet pressure and outlet pressure. Therefore, the pressure drop along pithe pe is inevitable and compressors are needed along pipelines to pump enough gas to satisfy downstream demand.

For a compressor with a capacity $C_{G_{-} t}$ and an annual growth rate $r_{G_{-} t}$ of gas flow on $t^{t h}$ pipeline, the current gas flow $q_{t}$ will take $n_{G_{-} t}$ years to reach compressor's capacity and reinforcement will be needed to ensure that future demand is met. Assuming that a CHP installed in the natural gas network at $s^{t h}$ node, where it is the node of coupling point $x^{\text {th }}$ with electricity network, gas flow change will appeal in the system. This will result in a new investment horizon and thus cause a different present value for the compressor investment, which gas network users need to pay in terms of network charges. 
Annuity factor is also applied to reflect time value of the investment. LRIC of the natural gas network is calculated by injecting unit size CHP to measure the change in future investment cost. The overall LRIC of natural gas network with a unit size CHP at node $s^{\text {th }}$ is

$$
L_{R I C_{G_{-} x}}=\sum_{t=1}^{T} \frac{\Delta P V_{G_{-} t}\left(G_{C H P}\right)}{G_{C H P} \times A F_{G_{-} t}}
$$

Where $G_{C H P}$ is gas input with the unit size 1MWe output CHP.

The LRIC for both networks with CHP sited at $x^{\text {th }}$ coupling point is the summation of $L R I C_{x_{-} E}$ and $L R I C_{x_{-} G}$ :

$$
L R I C_{x}=L R I C_{x_{-} E}+L R I C_{x_{-} G}
$$

LRIC index $I_{L R I C}$ in (1) shows the presumptive impact of CHP at each coupling point in both energy networks on the future reinforcement and network charges. Thus, the coupling point with minimum LRIC value indicates its economic feasibility for placing CHPs to reduce network investment and charges for customers.

\section{Calculation of Total Incremental Cost}

This section presents the objective function to determine the optimal size of the CHP. According to the LRIC index of CHP at each coupling point and the total number of CHPs to install, the power rating of the CHPs could be determined by minimising the total future network investment cost in both energy networks. In this case, the ratings of CHP becomes a variable instead of a fixed size.

The objective function of optimal CHP sizing is $(15)^{1}$. Where num $_{\text {CHP }}$ is the total number of CHP from 1 to Num, which should be smaller than the total number of the coupling point $X$. The change in energy flow in electricity and natural gas networks are caused by the electricity generation and gas consumption of one or several CHPs at different coupling points. The proposed LRIC method not only reflects the change of power and gas flow on the energy networks and their utilization rate, but more importantly, capture the economic benefits for both systems as CHPs can defer reinforcement horizon and reduce future investment costs when they are sited and sized appropriately.

\section{ELECTRICITY AND GAS NETWORK MODELLING}

\section{A. Electricity Network Modelling}

\section{1) Power Flow Formulation}

The power flow model is used to represent electricity network [28]. For a system with $M$ buses and $N$ branches, a branch $n$ connecting bus $i$ and bus $j$, the active power flow $P$ and reactive power flow $Q$ could be formulated in $(16)^{2}$.

\section{2) Bus Power Balance}

At each bus, the total power injected should be equal to the total outgoing power plus load, which applies to both active and reactive powers. By representing this power balance using power mismatch, equations are formulated in (17). The active and reactive power flow could be calculated using (18)

$$
\left\{\begin{array}{c}
\Delta P_{i}=P_{i}^{\text {Gen }}-P_{i}^{\text {Load }}-P_{i}^{\text {Flow }}=0 \\
\Delta Q_{i}=Q_{i}^{\text {Gen }}-Q_{i}^{\text {Load }}-Q_{i}^{\text {Flow }}=0
\end{array} \quad \forall i=1, \cdots, M\right.
$$

\section{3) Constraints}

To solve the power flow equation, per unit value is used to measure bus voltage and power. To maintain system security and reliability, constraints are set for voltage deviation:

$$
V_{m \_ \text {min }} \leq V_{m} \leq V_{m \_ \text {max }} \quad \forall m=1, \cdots, M
$$

Where the voltage limit is set between 0.94 to 1.06 per unit for a typical distribution network.

Normally, each branch also has its own capacity allowing maximum power flow:

$$
\left\{\begin{array}{l}
-P_{n}^{\text {Capa }} \leq P_{n} \leq P_{n}^{\text {Capa }} \\
-Q_{n}^{\text {Capa }} \leq Q_{n} \leq Q_{n}^{\text {Capa }}
\end{array} \quad \forall n=1, \cdots, N\right.
$$

$$
\begin{aligned}
& \min I C=\min \left(\sum_{n=1}^{N} \frac{\Delta P V_{E_{-} n}\left(E_{C H P}^{n u m}\right)}{E_{C H P}^{n u m} A F_{E_{-} n}}+\sum_{t=1}^{T} \frac{\Delta P V_{G_{-} t}\left(G_{C H P}^{n u m}\right)}{G_{C H P}^{\text {num }} A F_{G_{-} t}}\right) \quad \forall n u m=1, \cdots, N u m \\
& \left\{\begin{array}{c}
P_{n}=P_{i j}=V_{i}^{2}\left(\frac{G_{i j}}{a^{2}}+G_{s h i}\right)-\frac{V_{i} V_{j}}{a}\left(G_{i j} \cos \theta_{i j}+B_{i j} \sin \theta_{i j}\right) \\
Q_{n}=Q_{i j}=-V_{i}^{2}\left(\frac{B_{i j}}{a^{2}}+G_{s h i}\right)-\frac{V_{i} V_{j}}{a}\left(G_{i j} \sin \theta_{i j}-B_{i j} \cos \theta_{i j}\right)
\end{array} \quad \forall i, j=1, \cdots, M\right. \\
& \left\{\begin{array}{l}
P_{i}^{\text {Flow }}=\sum_{j=1}^{M, j \neq i}\left\{V_{i}^{2}\left(\frac{G_{i j}}{a^{2}}+G_{\text {shi }}\right)-\frac{V_{i} V_{j}}{a}\left(G_{i j} \cos \theta_{i j}+B_{i j} \sin \theta_{i j}\right)\right\} \\
Q_{i}^{\text {Flow }}=\sum_{j=1}^{M, j \neq i}\left\{-V_{i}^{2}\left(\frac{B_{i j}}{a^{2}}+G_{\text {shi }}\right)-\frac{V_{i} V_{j}}{a}\left(G_{i j} \sin \theta_{i j}-B_{i j} \cos \theta_{i j}\right)\right\}
\end{array} \quad \forall i=1, \cdots, M\right. \\
& q_{t}=q_{k l}=3.7435 \times 10^{-3} E f_{t}\left(\frac{T_{b}}{P_{b}}\right)\left(\frac{p_{k}^{2}-p_{l}^{2}}{g_{t} T_{f_{-} t} L_{t} Z_{t}}\right)^{0.5} D_{t}^{2.667} \quad \forall k, l=1, \cdots, S \\
& q_{k}^{\text {Flow }}=\sum_{l=1}^{S, l \neq k}\left\{3.7435 \times 10^{-3} E\left(\frac{T_{b}}{p_{b}}\right)\left(\frac{\operatorname{seg}(k, l)\left(p_{k}^{2}-p_{l}^{2}\right)}{g T_{f} L Z}\right)^{0.5} D^{2.667}\right\} \quad \forall k, l=1, \cdots, S
\end{aligned}
$$




\section{B. Natural Gas Network Modelling}

\section{1) Natural Gas Flow Formulation}

The gas flow model is used to represent the natural gas network [29]. For a natural gas network with $S$ nodes and $T$ pipelines, the gas flow in a pipeline $t$ between upstream node $k$ and downstream node $l$, without considering elevation difference between two nodes, is formulated using Weymouth Equation in (21).

\section{2) Node Gas Nodal Balance}

At each node, gas flow balance must be met in the gas infrastructure to assure that the total gas entering and injected must be equal to the total gas leaving and consumed at each node. This is represented by:

$$
\Delta q_{k}=q_{k}^{\text {In }}-q_{k}^{\text {Load }}-q_{k}^{\text {Flow }}=0 \quad \forall k=1, \cdots, T
$$

Where $\Delta q_{k}$ is the natural gas flow mismatch which should be 0 to satisfy nodal flow balance. $q_{k}^{\text {Flow }}$ is the sum of all the entering and leaving gas flows from an adjacent pipeline that connect to node $k$, represented in (23). $\operatorname{seg}(k, l)$ is 1 if node $k$ is the upstream node or otherwise $\operatorname{seg}(k, l)$ is -1 if node $l$ is the upstream node.

The calculation of natural gas flow is similar to that of power flow in an electricity network. Note that at least one node with specified node pressure must be given as a reference node in order to calculate the pressure of other nodes and thus calculate the gas flow among each pipeline.

\section{3) Constraints}

There are also some constraints of practical gas flow analysis. There is a gas flow constraint of each gas pipeline:

$$
q_{t_{-} \min } \leq q_{t} \leq q_{t_{-} \max } \quad \forall t=1, \cdots, T
$$

4) Variable Initialisation

The gas flow in (21) is dependent on the pressure difference of both sides of pipelines. However, this function could generate ill-conditioned elements in the Jacobian matrix if the pressure at each node is initialised as the same value. It might cause large or even null elements in the Jacobian matrix leading to a singular matrix. In order to avoid this case, the initialisation of gas node pressures at the upstream nodes is set $10 \%$ higher than the initialisation values of those at the downstream nodes. The slack node pressure is set as the reference value.

\section{THE MODELLING OF CHP AND COMPRESSOR STATION}

CHP is acting as a linkage between electricity and gas network. For a regional area supported by both electricity and gas networks, there will be many coupling or close points. Thus these coupling locations have the potential to integrate electricity and gas network with CHPs. In general, a gaspowered CHP model is expressed as follows:

$$
\left\{\begin{array}{c}
E_{C H P}=\eta_{E} G_{C H P} \\
H_{C H P}=\eta_{H} G_{C H P} \\
H t E R=\frac{\eta_{H}}{\eta_{E}}
\end{array}\right.
$$

Apparently, the insertion of CHP at a coupling point will cause energy flow changes in both networks. For the electricity network, CHP is acting as a local generator, fully or partially support the local demand, surplus electricity will be transferred to the grid.. For the natural gas network, CHP is acting as a load

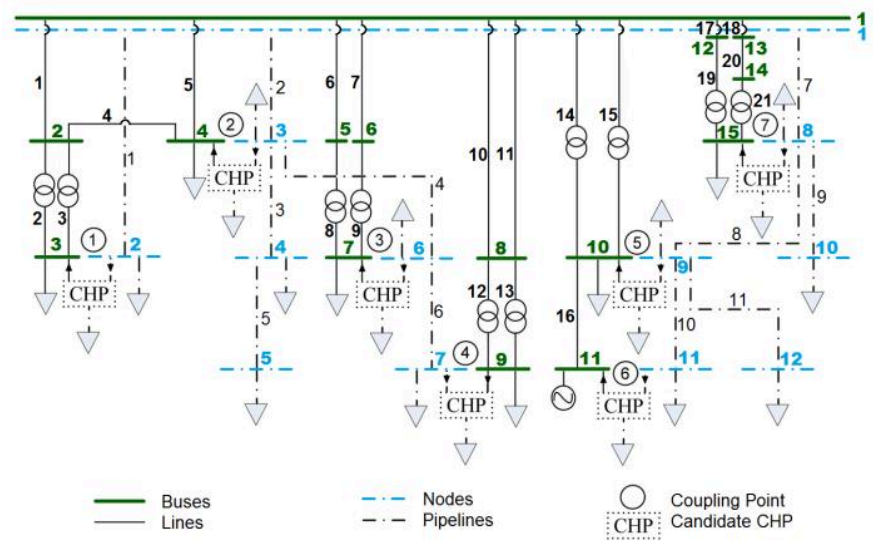

Fig. 2. An integrated electricity and gas systems with coupling points for CHP

to consume gas as the fuel and increase gas demand. Eventually, this will result in a change of network reinforcement horizon and consequently network investment costs.

For the electricity network, the incremental cost is to add parallel branches in case that any branch reaches capacity. For the natural gas network, the incremental cost is to add pressure compressors between nodes to assure enough pressure.

The work to isothermally compress $1 \mathrm{~kg}$ of natural gas for a compressor on pipeline $t$ between nodes $k$ and $l$ is:

$$
W i_{t}=\frac{286.76}{g_{t}} T_{f_{-} t} \log _{e}\left(\frac{p_{d_{-} t}}{p_{s_{-}}}\right) \quad \forall t=1, \cdots, T
$$

Where $T_{s}$ is suction the temperature of the gas, $p_{s}$ and $p_{d}$ are the suction and discharge pressure of the compressor that is equal to the upper and lower pressure limit $p_{\text {max }_{-} t}$ and $p_{\text {min_t }}$ of the pipeline.

The maximum gas flow along the $t^{\text {th }}$ pipeline is

$q_{\text {max } t}$

$=3.7435$

$\times 10^{-3} E f_{t}\left(\frac{T_{b}}{p_{b}}\right)\left(\frac{p_{\max _{-} t}^{2}-p_{\min _{-} t}^{2}}{g_{t} T_{f_{-} t} L_{t} Z_{t}}\right)^{0.5} D_{t}^{2.667}$

Thus the capacity of this compressor will be:

$$
C_{G_{-} t}=\frac{W i_{t} \times q_{\text {max }_{-} t}}{\eta_{t}}
$$

The final asset value of a compressor could be obtained:

$$
A V_{G_{-} t}=\left[\frac{C_{G_{-} t} /(24 * 3600)}{10^{6}}\right] \times 1341.02
$$

Where 1341.02 is the unit to convert MW into HP and 1520 is the capital price, in $£ / H P$ from [29].

\section{CASE STUdies}

An integrated electricity and gas system is analysed to demonstrate the proposed method. The potential coupling points are shown in Fig.2. The discount rate is set to 6.9\%, which is commonly used by the U.K.'s DNOs in setting network charges. A load growth rate of $1.6 \%$ per annum is taken from the project long-term investment statement in the U.K [30]. Two case studies are carried out on the integrated network to illustrate the performance of the proposed method in different scenarios: single CHP optimal planning, and multiple CHPs optimal planning. The electricity system comprises 15 buses, 
21 lines and 6 loads; while the natural gas system comprises 12 nodes, 11 branches and 11 loads. By considering the geographical distance between buses and nodes, a set of seven coupling points for candidate CHPs are considered accordingly.

\section{A. Single CHP Optimal Planning}

In this case, one single $\mathrm{CHP}$ is to be installed at the candidate coupling points.

\section{1) Optimal location}

Table I shows the results of LRIC value at each candidate location coupling by placing a unit size CHP. This unit CHP has Heat to Electricity Ratio (HtER) of 2 and overall efficiency of $72 \%$ according to the conventional CHP employed in the UK [3]. It has an electricity output of $1 \mathrm{MW}$ and heat output of $2 \mathrm{MW}$ and, thus its input should be $4.17 \mathrm{MW}$ equal to $0.10417 \mathrm{~m}^{3} / \mathrm{s}$ of gas. From this table, it could be revealed that the candidate CHP location with the lowest LRIC value is coupling point 1 , which links between Bus 3 and Node 2with a value of -3924 $£ /$ unit. This LRIC value is the sum of LRIC of the electricity network of $-3936 £ / M W$ and LRIC of the gas network of 12 $£ /\left(0.10417 \mathrm{~m}^{3} / \mathrm{s}\right)$. For electricity network, a $1 \mathrm{MW}$ injection of generation at Bus 3 will reduce $£ 3936$ of total network charge.

TABLE I

LRIC MATRIX FOR CHP CANDIDATE LOCATIONS BETWEEN TWO NETWORKS

\begin{tabular}{|c|c|c|c|c|c|c|c|}
\hline $\begin{array}{l}\text { Coup } \\
\text { Point }\end{array}$ & (1) & (2) & (3) & (4) & (5) & (6) & (7) \\
\hline \multicolumn{8}{|c|}{ Electricity line LRIC (£/MW) } \\
\hline Line & 3 & 4 & 7 & 9 & 10 & 11 & 15 \\
\hline 1 & -1690 & 1768 & -7 & -7 & -7 & -7 & -7 \\
\hline 2 & -146 & 0 & -1 & -1 & -1 & -1 & -1 \\
\hline 3 & -136 & 3 & 1 & 1 & 1 & 1 & 1 \\
\hline 4 & 0 & 0 & 0 & -234 & 0 & 0 & 0 \\
\hline 10 & 1 & 1 & 1 & -237 & 1 & 1 & 1 \\
\hline 11 & -1981 & -5637 & -8 & -8 & -8 & -8 & -8 \\
\hline 12 & -3 & -3 & -3 & -275 & -3 & -3 & -3 \\
\hline 13 & -2 & -2 & -2 & -273 & -2 & -2 & -2 \\
\hline 14 & 2 & 2 & 2 & 2 & -300 & -292 & 2 \\
\hline 15 & 2 & 2 & 2 & 2 & -224 & -217 & 2 \\
\hline 18 & 28 & 28 & 28 & 28 & 28 & 28 & -2766 \\
\hline$L R I C_{E}$ & -3936 & -3893 & 14 & -1002 & -514 & -499 & -2881 \\
\hline \multicolumn{8}{|c|}{ Natural gas pipeline LRIC $\left(£ /\left(0.10417 \mathrm{~m}^{3} / \mathrm{s}\right)\right)$} \\
\hline $\begin{array}{l}\text { Node } \\
\text { Branck }\end{array}$ & 2 & 3 & 6 & 7 & 9 & 11 & 8 \\
\hline 1 & 12 & 0 & 0 & 0 & 0 & 0 & 0 \\
\hline 2 & 0 & 208 & 208 & 208 & 0 & 0 & 0 \\
\hline 4 & 0 & 0 & 239 & 239 & 0 & 0 & 0 \\
\hline 6 & 0 & 0 & 0 & 28 & 0 & 0 & 0 \\
\hline 7 & 0 & 0 & 0 & 0 & 175 & 175 & 175 \\
\hline 8 & 0 & 0 & 0 & 0 & 75 & 78 & 0 \\
\hline$L R I C_{G}$ & 12 & 208 & 447 & 475 & 260 & 263 & 175 \\
\hline LRIC & -3924 & -3685 & 461 & -527 & -254 & -236 & -2706 \\
\hline
\end{tabular}

Table I also reveals the various LRIC change of network components following a CHP installation. For a CHP at coupling point 1 , the majority of LRIC reduction in electricity network is on line $1,2,3$ and 11 with a value of $-1690,-146$, 136 and $-1981 \mathrm{f} / \mathrm{MW}$, respectively. However, the power injection to the electricity network through CHP will cause $0.1042 \mathrm{~m}^{3} / \mathrm{s}$ of gas load increase at node 2 , which results in an increase in gas network charge by $£ 12$ on pipeline 1 . By combining network charges in both networks, it could be seen that coupling point 1 between electricity bus 3 and gas node 2 is considered to be the optimal site for single CHP planning.

\section{2) Optimal capacity}

From the first step, the optimal site of CHP is at coupling point 1 . The optimal size of the CHP placed at this point will be determined by calculating the lowest incremental cost (IC) that the CHP could benefit both networks. For practical reason, the maximum electrical capacity of CHP is limited at $25 \mathrm{MW}$. The results of single CHP optimal planning are in Table II.

TABLE II

Future INVESTMENT COST CHANGE FOR CHP CONNECTED CHOSEN SiTE

\begin{tabular}{cccccc}
\hline \hline No & $\begin{array}{c}\text { E-network } \\
\text { Average } \\
\text { Reinforcement } \\
\text { horizon (year): }\end{array}$ & 94.8 & $\begin{array}{c}\text { G-network } \\
\text { Average } \\
\text { Reinforcement } \\
\text { horizon (year): }\end{array}$ & 88.4 & \\
\hline CHP & $\begin{array}{c}\text { Average } \\
\text { Reinforcement }\end{array}$ & E IC & $\begin{array}{c}\text { Average } \\
\text { Reinforcement } \\
\text { horizon deferral } \\
\text { size }\end{array}$ & G IC & Total \\
$(\mathrm{MW})$ & $\begin{array}{c}\text { horizon deferral } \\
\text { (year) }\end{array}$ & $(\mathrm{k} £)$ & IC (kf) \\
\hline 1 & -0.6 & -3.9 & 1.0 & 0.01 & -3.9 \\
5 & -3.1 & -16.8 & 3.8 & 0.17 & -16.6 \\
10 & -7.2 & -25.7 & 6.1 & 0.95 & 24.8 \\
15 & -15.2 & -33.6 & 7.7 & 3.1 & -30.4 \\
16 & -22.2 & -34.4 & 8.0 & 3.9 & -30.5 \\
20 & -15.6 & -36.9 & 9.0 & 8.0 & -28.9 \\
25 & -20.2 & -38.4 & 10.0 & 17.0 & -21.4 \\
\hline \hline
\end{tabular}

TABLE III

RESULTS OF SINGLE CHP PLANNING

\begin{tabular}{|c|c|c|c|c|c|c|c|}
\hline \multicolumn{4}{|c|}{ Coupling Location } & \multicolumn{4}{|c|}{1} \\
\hline \multicolumn{4}{|c|}{ Electricity Bus } & \multicolumn{4}{|c|}{3} \\
\hline \multicolumn{4}{|c|}{ Gas Node } & \multicolumn{4}{|c|}{2} \\
\hline \multicolumn{4}{|c|}{$\mathrm{E} / \mathrm{G}[\mathrm{MW}]$} & \multicolumn{4}{|c|}{$16 / 67$} \\
\hline \multicolumn{4}{|c|}{ Objective (Total IC) [k£] } & \multicolumn{4}{|c|}{-30.6} \\
\hline \multicolumn{4}{|c|}{ Electricity network } & \multicolumn{4}{|c|}{ Natural gas network } \\
\hline $\mathrm{L}$ & $\mathrm{UC}$ & RHD & IC & $\mathrm{P}$ & $\mathrm{UC}$ & RHD & IC \\
\hline 1 & -25.2 & 30.7 & -15.0 & 1 & 47 & -87.7 & 3.85 \\
\hline 2 & -25.3 & 52.3 & -1.0 & & & & \\
\hline 3 & -24.9 & 52.2 & -0.9 & & & & \\
\hline 5 & -22.3 & 299 & -0.04 & & & & \\
\hline 11 & -25.4 & 31.7 & -17.5 & & & & \\
\hline & -5.9 & 22.2 & -34.4 & & 4.3 & -8.0 & 3.85 \\
\hline
\end{tabular}

L: electricity line; P: gas pipeline; UC: Utilization change (\%); RHD: Reinforcement horizon deferral (year); IC: Incremental cost (kf)

The IC reaches the minimum at $£-30.5 \mathrm{k}$ with the CHP's electrical capacity at $16 \mathrm{MW}$ and a total capacity of $67 \mathrm{MW}$. With the determined CHP size and location, an electricity network future investment reduction of $£-34.4 \mathrm{k}$ is achieved, with the electricity network reinforcement deferral of 22.2 years. Although gas network future investment increases by 33.9k with a short future reinforcement horizon by 8 years, the total investment costs for the integrated energy systems are optimised. Ideally, $16 \mathrm{MW}$ electricity output is equivalent to 66.67 MW gas input. In practice, CHP is generally sized in integer thus the capacity of the CHP is selected as $67 \mathrm{MW}$ with an electricity output of $16 \mathrm{MW}$.

By ascending the capacity of the CHP, the changing trend of the incremental cost and future investment horizon for both networks are shown in Table II. Before installing CHP, their respective average future reinforcement horizon is 94.8 years for electricity network and 88.4 years for the gas network. By increasing CHP capacity from 0 to $67 \mathrm{MW}$, it is seen that the incremental cost for the electricity network drops continuously 
and the average reinforcement horizon deferral increase, indicating with increasing CHP capacity, transmission utilization decreases and defers the future investment horizon, consequently reducing the network charges. However, the situation is totally opposite on the gas network as CHP consumes gas to generate electricity and heating. Thus gas network charge will increase as the incremental cost increases and the time for future investment is shortened. The overall incremental cost for both networks reaches a minimum with CHP capacity 16MW.

Table III shows the detailed comparison of line capacity utilization, future reinforcement horizon and present value before and after CHP installation. At the optimal size of 67MW with an electricity output of $16 \mathrm{MW}$, power utilization on lines $1,2,3,5$ and 11 are reduced by all around $25 \%$. Their future investment horizons are deferred by 31, 52, 52399 and 32 years respectively. The total difference of electricity network present value for all lines is $£-496,735$. By taking the annuity factor into account the total incremental cost reduction would be $£$ 34,440 . As for the gas network, significant growth of gas flow is added on pipeline 1 as the CHP consumes gas. This also results in a reduced future investment horizon from 117 years to 30 years. The change of the present value of this pipeline is $£ 55,577$, equivalent to $£ 3853$ of incremental cost. The objective function is $£-30,587$. Thus, the optimized site to install is at the coupling point between Bus 2 and Node 3 with an optimal size of $67 \mathrm{MW}$, which brings a total IC reduction of $£ 30.6 \mathrm{k}$.

\section{B. Multiple CHPs Optimal Planning}

In this case study, multiple CHPs of three are assumed to be installed on the same energy networks.

\section{1) Optimal locations}

According to the LRIC matrix results in Table I, the optimal sites for the three identical CHPs are coupling points 1,2 , and 7, with respective LRIC value of $£-3,924, £-3,685$ and $£-2,706$ per MW. Thus, these three locations are selected as the optimal coupling sites between two energy networks to install CHPs.

\section{2) Optimal capacities}

The optimal size of each CHP and the change of network load utilization, future reinforcement deferral and total incremental cost are shown in Table IV. The optimal capacities of the CHPs at coupling points 1,2 and 7 are 54.17, 37.5 and 41.67 MW with a respective electricity output of 13,9 and 10 MW. The objective (total IC) is $£-45,224$ comprised of an electricity network incremental cost of $£-53,867$ and a natural gas network incremental cost of $£ 8,643$.

For the electricity network, the line utilization has been changed significantly with the CHP, especially on lines $1,2,3$, 11 and 18 with a utilization reduction of $35.3 \%, 20.6 \%, 20.3 \%$, $28.2 \%$ and $23.5 \%$, respectively. The resultant average future reinforcement horizon deferral of the electricity network is 29.8 years with an overall present value difference of $£-776,922$. By considering the annuity factor, the overall electricity network incremental cost reduction is $£-53,867$.

For the natural gas network, the load at the node which locates CHP increases greatly and results in increased utilization of related gas pipeline. In this case, there is a great amount of gas flow increase on pipeline 1,2 and 7 with the
TABLE IV

Results OF MultiPle CHP PlanNING (3 CHPs)

\begin{tabular}{|c|c|c|c|c|c|c|c|}
\hline \multicolumn{2}{|c|}{ Location } & \multicolumn{2}{|c|}{1} & \multicolumn{2}{|c|}{2} & \multicolumn{2}{|c|}{7} \\
\hline \multicolumn{2}{|c|}{ Bus } & \multicolumn{2}{|c|}{3} & \multicolumn{2}{|c|}{4} & \multicolumn{2}{|c|}{15} \\
\hline \multicolumn{2}{|c|}{ Node } & \multicolumn{2}{|c|}{2} & \multicolumn{2}{|c|}{3} & \multicolumn{2}{|c|}{8} \\
\hline \multirow{2}{*}{\multicolumn{2}{|c|}{$\begin{array}{c}\text { E/G [MW] } \\
\text { Objective } \\
\text { (Total IC) [k£] }\end{array}$}} & \multicolumn{2}{|c|}{$13 / 54$} & \multicolumn{2}{|c|}{$9 / 38$} & \multicolumn{2}{|c|}{$10 / 42$} \\
\hline & & & & \multicolumn{2}{|c|}{-45.2} & & \\
\hline \multicolumn{4}{|c|}{ Electricity network } & \multicolumn{4}{|c|}{ Natural gas network } \\
\hline $\mathrm{L}$ & $\mathrm{UC}$ & RHD & $\mathrm{IC}$ & $\mathrm{P}$ & $\mathrm{UC}$ & RHD & IC \\
\hline 1 & -35.3 & 49.0 & -16.5 & 1 & 38.2 & 78.2 & 2.03 \\
\hline 2 & -20.6 & 38.7 & -9.6 & 2 & 10.6 & 14.2 & 2.78 \\
\hline 3 & -20.3 & 38.6 & -9.1 & 7 & 21.3 & 27.5 & 3.84 \\
\hline 5 & -9.1 & 32.5 & -0.04 & & & & \\
\hline 6 & -15.4 & 66.4 & -0.07 & & & & \\
\hline 11 & -28.2 & 36.3 & -18.1 & & & & \\
\hline 17 & -15.9 & 76.0 & -0.24 & & & & \\
\hline 18 & -23.5 & 30.3 & -16.9 & & & & \\
\hline 19 & -15.9 & 101 & -0.03 & & & & \\
\hline 21 & -15.3 & 129 & -0.02 & & & & \\
\hline & -9.5 & 29.8 & -53.9 & & 6.4 & 15.8 & 9.64 \\
\hline
\end{tabular}

L: electricity line; P: gas pipeline; UC: Utilization change (\%); RHD: Reinforcement horizon deferral (year); IC: Incremental cost (k£)

utilization growth of $38.2 \%, 10.6 \%$ and $21.3 \%$, respectively. This growth causes the horizon of pipeline reinforcement shortened by 78,14 and 27 years. The total present value difference and overall incremental cost between no CHP and with multi-CHP scenarios are $£ 124,663$ and $£ 8,643$, respectively. Thus, the total network charge will be the sum of the IC of both networks, which is $£-45,224$.

\section{Comparative with the State-of-the-Art}

In this section, a comparison is conducted between the proposed method and other CHP planning study with a different objective. The test system is the same as that in the previous case and the objective of other study is obtained from [6,31]. The objective function is to minimise the total investment costs of CHP, modelled by:

$$
\min O F=C_{t}^{I M O}+C_{t}^{E N S}+C_{t}^{\text {loss }}+C_{t}^{V P F}
$$

Where $C_{t}^{I M O}$ is the cost including installation, maintenance and operation, $C_{t}^{E N S}$ is the network reliability cost, $C_{t}^{\text {loss }}$ is the power loss cost and $C_{t}^{V P F}$ is the voltage penalty cost.

The detailed modelling of each cost is represented mathematically in [6]. In this case, it is used to evaluate how the location and capacities of CHP would change with a different objective in the same energy system. Three CHPs are supposed to be installed in the energy system and comparisons are conducted with the results in Case B.

By applying the optimum solver using the interior-point method the results of CHPs' location and capacity with the minimised objective are in Table V. They are respectively located at coupling point 4 between bus 9 and node 7 , coupling point 6 between bus 11 and node 11 and coupling point 7 between bus 15 and node 8 , with an electricity/gas capacity of $24 / 100,8 / 33$ and $18 / 75 \mathrm{MW}$. It can be observed that the total cost of the objective is decreased from $£ 355.66 \mathrm{M}$ to $£ 345.29 \mathrm{M}$ by $2.9 \%$ after the CHPs optimally placed and sized. The biggest reduction is on the operation cost with a value of $£ 51.62 \mathrm{M}$. This is due to the replacement of primary energy of CHP by using more gas and generating electricity and heat. Network 
reliability cost is also significantly reduced from $£ 102.67 \mathrm{M}$ to $£ 80.61 \mathrm{M}$ by $21.5 \%$ that using more gas will beneficial to the electricity network. Power loss cost is reduced by $26.4 \%$ to $£ 0.92 \mathrm{M}$. The main reason for the reduction is the decrease in purchasing grid electricity for supplying electrical demand as a result of CHPs in the network. The installation cost is $£ 45.55 \mathrm{M}$ and the maintenance cost is $£ 18.25 \mathrm{M}$. It is also known that the total cost savings before and after CHP planning are $£ 10.27 \mathrm{M}$. Most of the reduction is from operation cost and network reliability cost, while the installation and maintenance cost for CHP compensate part saving.

By comparing Table IV and V, it could be concluded that when the planning objective is different, there is a big change in both the optimal location and capacity of CHP in the same network. While the study in [6] is focused on the benefits of optimal CHP planning considering current network reliability, power loss and voltage profile. However, the method proposed in this paper is focused on the reducing of future network investment and reinforcement and thus use-of-system charges.

\section{DISCUSSION}

This paper is focused on CHP planning to reduce network investment and UoS charges, considering its impacts on both electricity and gas network. This new planning is very different from other existing research, which mainly focuses on energy cost, carbon reduction or supply reliability. The model in this paper conducts a forward-looking planning model in terms of future reinforcement deferral, investment cost reduction and the UoS charge reduction. The method will determine the optimal locations and ratings of CHPs that benefit not only themselves but also network users. However, as demonstrated in the case study, the planning results could be very different if the objectives are different. Thus, it is hard to tell which method is better. However, to incorporate other objectives, such as costs, emission reductions, the models will be much complicated.

The future work will be carried out in two aspects. Firstly, as CHP is operating to provide both electricity and heating, the heating network is reasonable to be added into the study. Secondly, improving the modelling of CHP is critical to recognise the dynamic characteristics of the energy conversions and reflect the impact on particularly natural gas systems.

\section{CONCLUSION}

This paper proposes a novel approach for the optimal planning of CHP in a multi-carrier energy system by considering CHP's impact on future network reinforcement and network charges. It aims to reduce network investment costs and thus could bring potential benefits to network owners and users. Through extensive case study and comparison, the following observations are obtained.

- LRIC matrix index is an effective indicator to determine the optimal location and capacity of CHP in an integrated electricity and gas networks.

- It also shows that CHP planning in the integrated network would significantly reduce the incremental cost for the electricity network and slightly increase the incremental cost of the gas network.
- The comparison with state-of-the-art indicates that when a different objective is applied, the optimal locations and capacities of CHP changes accordingly, indicating the importance of setting a proper planning objective.

- Overall, the case study illustrates that the proposed LRIC method for CHP planning can enable future network reinforcement deferral and consequently reduce the incremental cost of network investment.

\section{REFERENCES}

[1] N. Jenkins, J. Ekanayake, and G. Strbac, Distributed Generation. Institution of Engineering and Technology, 2010.

[2] (22 January 2013). Guidance Combined heat and power. Available: https://www.gov.uk/guidance/combined-heat-and-power

[3] (2017). Digest of UK Energy Statistics (DUKES). Available: https://assets.publishing.service.gov.uk/government/uploads/system/uploa ds/attachment_data/file/643414/DUKES_2017.pdf

[4] A. K. Basu, A. Bhattacharya, S. Chowdhury, and S. P. Chowdhury, "Planned Scheduling for Economic Power Sharing in a CHP-Based MicroGrid," IEEE Transactions on Power Systems, vol. 27, no. 1, pp. 30-38, 2012.

[5] C. Wu, W. Gu, P. Jiang, Z. Li, H. Cai, and B. Li, "Combined Economic Dispatch Considering the Time-Delay of District Heating Network and Multi-Regional Indoor Temperature Control," IEEE Transactions on Sustainable Energy, vol. 9, no. 1, pp. 118-127, 2018.

[6] S. Pazouki, A. Mohsenzadeh, S. Ardalan, and M. R. Haghifam, "Optimal place, size, and operation of combined heat and power in multi-carrier energy networks considering network reliability, power loss, and voltage profile," IET Generation, Transmission \& Distribution, vol. 10, no. 7, pp. 1615-1621, 2016.

[7] X. Zhang, M. Shahidehpour, A. Alabdulwahab, and A. Abusorrah, "Optimal Expansion Planning of Energy Hub With Multiple Energy Infrastructures," IEEE Transactions on Smart Grid, vol. 6, no. 5, pp. 2302$2311,2015$.

[8] D. Gvozdenac, B. G. Urošević, C. Menke, D. Urošević, and A. Bangviwat, "High-efficiency cogeneration: CHP and non-CHP energy," Energy, vol. 135, pp. 269-278, 2017/09/15/2017.

[9] A. Gimelli et al., "Optimal configuration of modular cogeneration plants integrated by a battery energy storage system providing peak shaving service," Applied Energy, vol. 242, pp. 974-993, 2019/05/15/ 2019.

[10]X. Zhang, G. G. Karady, and S. T. Ariaratnam, "Optimal Allocation of CHP-Based Distributed Generation on Urban Energy Distribution Networks," IEEE Transactions on Sustainable Energy, vol. 5, no. 1, pp. 246-253, 2014.

[11]S. Chen, Z. Wei, G. Sun, K. W. Cheung, and Y. Sun, "Multi-Linear Probabilistic Energy Flow Analysis of Integrated Electrical and NaturalGas Systems," IEEE Transactions on Power Systems, vol. 32, no. 3, pp. 1970-1979, 2017.

[12]S. Clegg and P. Mancarella, "Integrated Electrical and Gas Network Flexibility Assessment in Low-Carbon Multi-Energy Systems," IEEE Transactions on Sustainable Energy, vol. 7, no. 2, pp. 718-731, 2016.

[13]J. Wang et al., "Economic Benefits of Integrating Solar-Powered Heat Pumps Into a CHP System," IEEE Transactions on Sustainable Energy, vol. 9, no. 4, pp. 1702-1712, 2018.

[14]H. Wang, W. Yin, E. Abdollahi, R. Lahdelma, and W. Jiao, "Modelling and optimization of CHP based district heating system with renewable energy production and energy storage," Applied Energy, vol. 159, pp. 401$421,2015 / 12 / 01 / 2015$.

[15]O. A. Shaneb, G. Coates, and P. C. Taylor, "Sizing of residential $\mu$ CHP systems," Energy and Buildings, vol. 43, no. 8, pp. 1991-2001, 2011/08/01/ 2011.

[16]H. Li, Z. Lu, Y. Qiao, and N. Wang, "A Non-Sequential Probabilistic Production Simulation Method for Wind Energy Curtailment Evaluation Considering the Seasonal Heat Supply Constraints," IEEE Transactions on Sustainable Energy, vol. 9, no. 1, pp. 462-473, 2018.

[17]S. Moradi, R. Ghaffarpour, A. M. Ranjbar, and B. Mozaffari, "Optimal integrated sizing and planning of hubs with midsize/large CHP units considering reliability of supply," Energy Conversion and Management, vol. 148, pp. 974-992, 2017/09/15/2017.

[18]M. Quashie, C. Marnay, F. Bouffard, and G. Joós, "Optimal planning of microgrid power and operating reserve capacity," Applied Energy, vol. 210, pp. 1229-1236, 2018/01/15/2018. 
[19]N. Gird, "Electricity Transmission network charging," 2018, Available: https://www.nationalgrid.com/sites/default/files/documents/Introduction \%20to\%20TNUoS\%2C\%20BSUoS\%20and\%20Connection\%20Charging .pdf.

[20] J. Li, C. Yuan, and F. Li, "The relationship of constraints cost and load factor: A evaluation for the improved ICRP method," in 2013 10th International Conference on the European Energy Market (EEM), 2013, pp. 1-8.

[21]G. Strbac and J. Mutale, "Framework and methodology for pricing of distribution networks with distributed generation," Cent. Distrib. Gener. Sustain. Electr. Energy, 2005.

[22]F. Li, "Long-Run Marginal Cost Pricing Based on Network Spare Capacity," IEEE Transactions on Power Systems, vol. 22, no. 2, pp. 885886, 2007.

[23]F. Li and D. L. Tolley, "Long-Run Incremental Cost Pricing Based on Unused Capacity," IEEE Transactions on Power Systems, vol. 22, no. 4 , pp. 1683-1689, 2007.

[24]C. Gu, W. Yang, Y. Song, and F. Li, "Distribution Network Pricing for Uncertain Load Growth Using Fuzzy Set Theory," IEEE Transactions on Smart Grid, vol. 7, no. 4, pp. 1932-1940, 2016.

[25]C. Williams and K. Elmhirst, "Gas Transmission Transportation Charges," 12 Jan 2018.

[26] Ofgem. (2018, 13 Jun 2018). Charging arrangements.

[27]X. Yan, X. Yang, H. Wang, C. Gu, and F. Li, "Pricing for integrated electricity and gas systems based on long-run incremental cost," in 2017 IEEE Power \& Energy Society General Meeting, 2017, pp. 1-5.

[28]H. Saadat, Power System Analysis. McGraw-Hill, 2009.

[29]E. S. Menon, Gas Pipeline Hydraulics. CRC Press, 2005.

[30]N. Grid, "National Electricity Transmission System Seven Year Statement," May 2010, $\quad$ Available: www2.nationalgrid.com/WorkArea/DownloadAsset.aspx?id=43281.

[31] S. Pazouki, A. Mohsenzadeh, S. Ardalan, and M. Haghifam, "Optimal place, size, and operation of combined heat and power in multi carrier energy networks considering network reliability, power loss, and voltage profile," IET Generation, Transmission \& Distribution, vol. 10, no. 7, pp. 1615-1621, 2016. 